\title{
Surgical Repair of a Ruptured Giant Abdominal Aortic Aneurysm in a 16-Year-0ld with Takayasu's Arteritis: Case Report and Etiological Review
}

José Maciel Caldas dos Reis, ${ }^{1 \oplus}$ Glauco dos Santos Melo, ${ }^{1}$ Murilo Vasconcelos de Oliveira, ${ }^{\circledR}$ Felipe Eduardo de Oliveira Santos, ${ }^{2}{ }^{\circledR}$ Tereza Maria Meireles Fernandes da Silva, ${ }^{\circledR}{ }^{\circledR}$ Hugo Luis da Silva Ferreira ${ }^{4}{ }^{\complement}$

Division of Vascular and Endovascular Surgery at Gaspar Vianna Clinics Hospital (HCGV), ${ }^{1}$ Belém, PA - Brazil

Division of General Surgery at Gaspar Vianna Clinics Hospital (HCGV), ${ }^{2}$ Belém, PA - Brazil

Pará State University (UEPa), Belém, PA - Brazil

Federal University of Pará (UFPa), ${ }^{4}$ Belém, PA - Brazil

\begin{abstract}
Takayasu's arteritis is a type of primary systemic vasculitis that affects medium and large arteries, including the aorta and its main branches, as well as the pulmonary and coronary arteries. Although rare in children, it is the third most common vasculitis in the pediatric population, often with delayed diagnosis due to the nonspecific presentation of clinical symptoms in its initial phase. This is a case of a 16-year-old girl with a giant ruptured abdominal aortic aneurysm, who needed surgery on an emergency basis. The etiological aspects involved in aneurysms in young patients are also addressed.
\end{abstract}

\section{Introduction}

Abdominal aortic aneurysms (AAAs) are rare in the pediatric population. ${ }^{1}$ They require high clinical suspicion, since they remain asymptomatic for a long period time, and, much like in adults, they also present a serious risk of rupture and death. ${ }^{1,2}$ These AAAs have also been associated with systemic diseases, developmental abnormalities, and connective tissue disorders. ${ }^{2,3}$

Takayasu's arteritis (TA) is a chronic progressive inflammatory disease characterized by granulomatous vasculitis, which involves the aorta and its main

\section{Keywords}

Aortic Aneurysm Abdominal/surgery; Takayasu Arteritis; Vasculitis; Young Adult; Child; Mortality. branches. ${ }^{3}$ Granulomatous transmural inflammation may eventually lead to stenosis or occlusions in the involved vessels. Though aneurysms and aortic dissections are rare events, particularly in the pediatric population and young adults, they are now more frequently being diagnosed. However, TA diagnosis remains a major challenge due to its nonspecific clinical and laboratory features.,

An aneurysm is defined by the Society of Vascular Surgery as a localized, permanent dilation of an artery with at least a $50 \%$ increase in diameter size as compared to the expected normal diameter of the artery or its proximal segment..$^{5-7}$ AAAs can be classified according to their relative location to the renal arteries, morphology, diameter, or etiology, and are three to eleven times more common in men than in women. ${ }^{5-7}$

A ruptured AAA is a major life event that carries a high mortality rate. ${ }^{7}$ Immediate diagnosis and treatment is essential to improve the outcome because of its time-sensitive nature. ${ }^{7,8}$

Emergency physicians should maintain a higher rate of suspicion for prompt diagnosis of aortic aneurysm in any individual regardless of age when presented with such symptoms as abdominal pain, especially if associated with hypertension and radiating back pain. ${ }^{8,9}$

The case presented herein is of particular interest, reporting on a 16-year-old girl with TA who had a ruptured giant abdominal aortic aneurysm. The etiological aspects involved in aneurysms in this population will be reviewed and discussed. 


\section{Case presentation}

The patient was a 16-year-old female with hypertension, who had suffered episodes of palpitations and hypertensive crises over the past two years.

The patient was admitted to the emergency room of the hospital with a sudden onset of abdominal pain, associated with skin and mucosal pallor and back pain. Her pain had begun at home 4 hours before being admitted to the hospital.

The patient had been attending regular nephrological and cardiological follow-up consultations at the institution due to difficult-to-control hypertension and deterioration of renal function. She was also using taking presciption medications and was not a smoker or illicit drug user. There was no family history of aneurysm and no report of trauma, diarrhea, constipation, fever, or symptoms of dysuria or hematuria. She was $1.65 \mathrm{~m}$ tall, with a body mass index (BMI) of $24 \mathrm{~kg} / \mathrm{m}^{2}$. There were no indications of connective tissue disease with arterial involvement, such as hyperelastic skin, hypermobile joints, or Marfanoid habitus. She reported that 3 months ago she had received the diagnosis of TA, based on clinical and tomographic criteria.

At the initial presentation and physical examination, the patient was experiencing intense abdominal pain. Diffuse abdominal tenderness to palpation was observed, mainly in the lower abdominal region, and the presence of a pulsatile mass in the epigastrium and mesogastrium regions during the abdominal physical examination. She reported that the pain radiated to her back, preventing active movements in the left lower limbs. Her vital signs were stable upon admission, with a heart rate of $115 \mathrm{bpm}$ and a blood pressure of 100/60 $\mathrm{mmHg}$.

Emergency laboratory tests revealed abnormal concentration levels of hemoglobin, $6.2 \mathrm{~g} / \mathrm{dL}$; hematocrit, $21 \%$; leukocytes, $12,000 / \mathrm{mm}^{3}$; platelets, $180,000 / \mathrm{mm}^{3}$; Urea, 76; and Creatinine, 2.1. An electrocardiogram showed signs of left ventricular hypertrophy.

A ruptured abdominal aortic aneurysm was suspected, and the patient was immediately referred to the radiology ward, where she underwent an abdominal CT angiography before being transferred to the operating room.

The CT angiography revealed a ruptured abdominal infrarenal aortic aneurysm, which opened to the retroperitoneum, with a maximum estimated diameter of $11.0 \mathrm{~cm}$ (Figure 1). The site of the distal aortic rupture and the bilateral ostial occlusion of the renal artery stood out in the $\mathrm{CT}$ angiogram image reconstruction (Figure 2).

In the emergency surgery, a xiphopubic midline abdominal incision was made, the mesentery was exposed, and the intestines were immobilized. Transperitoneal dissection along the ligament of Treitz revealed a giant aneurysm. It was carefully dissected and found to be ruptured in its posterolateral wall on the left, $3 \mathrm{~cm}$ above the bifurcation to the iliac arteries, with a significant retroperitoneal hematoma (Figure 3 ).

The infrarenal aorta and the common iliac artery were dissected for repairs and to apply clamps. The proximal aorta and both iliac arteries were clamped after systemic heparinization.

The aneurysm was opened and the mural thrombus removed. The bifurcation of the juxtarenal aorta and both common iliac arteries presented no aneurysmatic alterations and were used for distal control. As the patient presented no clinical evidence or operative findings compatible with active aortic infection, there was minimal concern with the placement of the prosthetic material. A $20 \times 10 \mathrm{~mm}$ Dacron graft was used for a bilateral aortoiliac graft using 2-0 polypropylene thread in a continuous suture (Figure 4). The aneurysm sac was sutured over the graft with no leaks. After two days in the intensive care unit, the patient was transferred to the general ward. While in the intensive care unit, she underwent renal replacement therapy with hemodialysis by a catheter inserted into the common femoral vein. She had a total of six sessions of hemodialysis until she was stable and conservative treatment was resumed. The patient was discharged from the hospital on the seventh postoperative day and the follow-up at six months was normal.

Tomographic and intraoperative findings were compatible with the presence of a true aneurysm. The aneurysm walls were thicker than that of the normal aorta. A small fragment from the mural thrombus and the aorta were sent for culture and histopathological analysis. A histopathological examination of the aneurysm with conventional hematoxylin-eosin staining revealed that the aneurysm wall had a disordered structure with nonspecific inflammation in the tunica media and adventitia that was comparable to TA. Both the aneurysm and the thrombus wall cultures were negative for bacteria. 


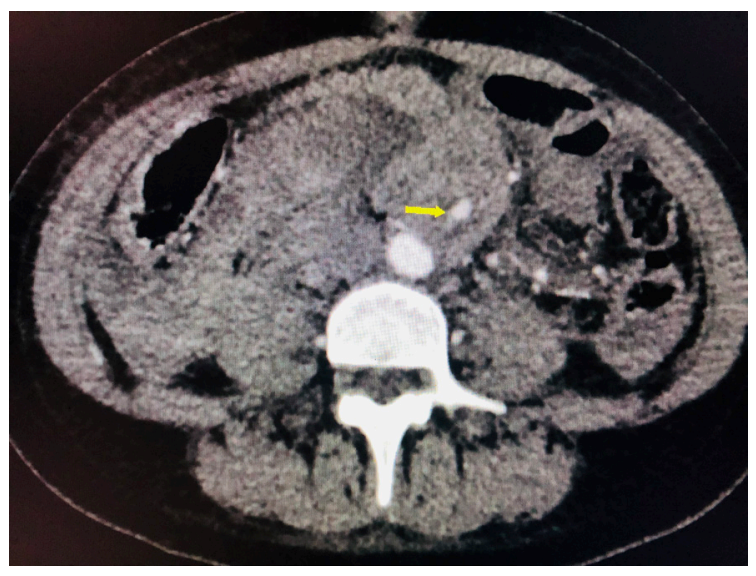

Figure 1 - Coronal CT angiogram images showing a ruptured giant aneurysm.

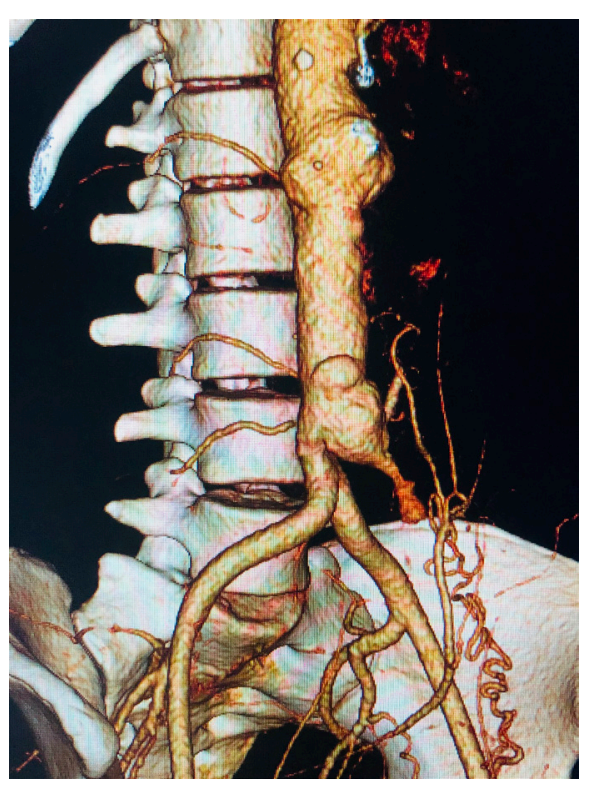

Figure 2. Three-dimensional CT angiogram showing a distal aortic rupture and the bilateral ostial occlusion of the renal artery.

\section{Discussion}

The true incidence of aneurysms in Brazil is unknown. Studies in several European countries and in the United States estimate the prevalence of AAA to be between $4.2 \%$ and $8.8 \%$ and between $0.6 \%$ and $1.4 \%$ for men and women over 50 years of age, respectively. ${ }^{1,3,7}$ The most common etiologies include atherosclerosis, but aneurysms can also be secondary responses to syphilis, trauma, and congenital and inflammatory arteritis (Takayasu, Horton, and Kawasaki diseases). ${ }^{2-7}$ Symptoms are often nonspecific during the early stages, which can result in diagnostic delays. ${ }^{3,4}$

The literature on pediatric aneurysm is limited to reports and small case series. ${ }^{1}$ The etiology, natural progression, and prognosis of aortic aneurysms in children and young adults remain unknown, due to their rarity. ${ }^{1,2}$ When present, this type of aneurysm is usually associated with underlying pathological processes or systemic diseases. ${ }^{3,4}$ 


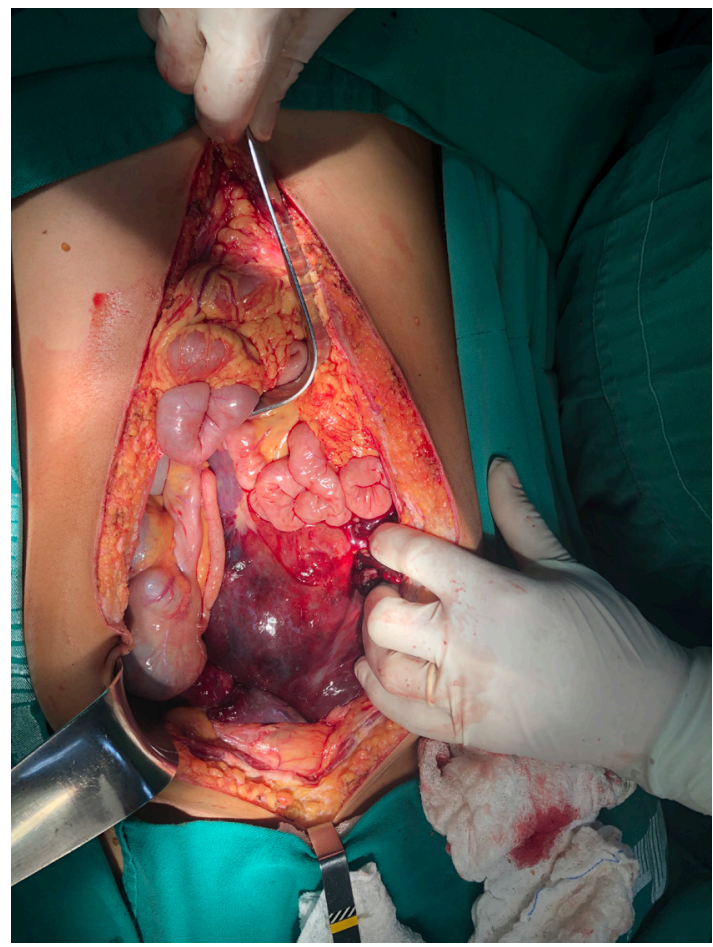

Figure 3 - Intraoperative finding of a giant retroperitoneum hematoma.

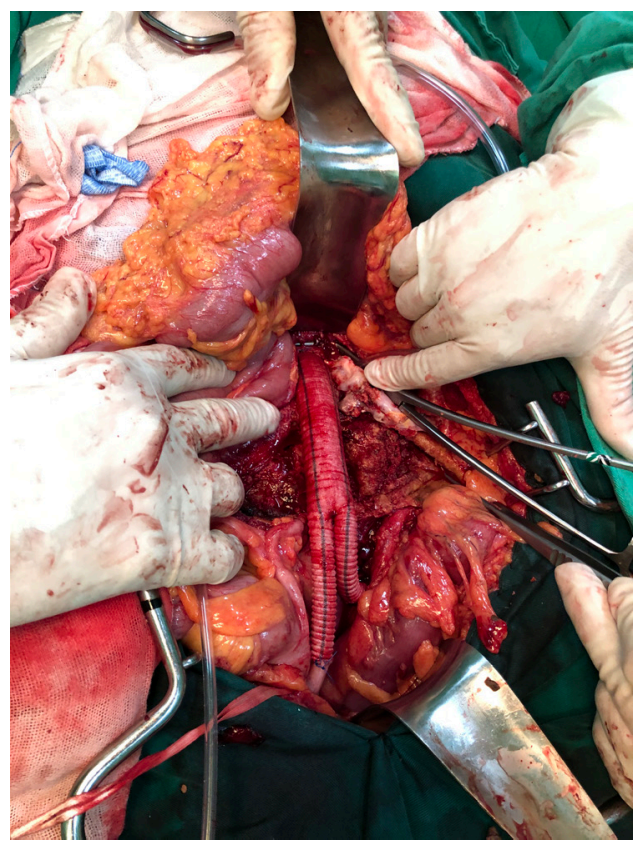

Figure 4 - Dacron graft used for bilateral aortoiliac reconstruction. 
Patients with TA are mostly female with hypertension, which at times is difficult to control, together with other nonspecific symptoms, such as headache, nausea, and palpitations. The association of these symptoms with aneurysmal aortic injury is rarely observed, but it should always be considered for differential diagnosis., ${ }^{4,10}$

Whatever the cause and location, aortic aneurysms are life-threatening due to the risk of rupture. ${ }^{1-4}$ In our patient's case, systemic arterial hypertension, which had been diagnosed 2 years prior, was the associated cardiovascular risk factor.

The differential diagnosis of TA includes other causes of large-vessel vasculitis, such as inflammatory aortitis (syphilis, tuberculosis, lupus, rheumatoid arthritis, spondyloarthropathies, Kawasaki's disease, and giant-cell arteritis), developmental abnormalities (aortic coarctation and Marfan's syndrome), and aortic pathologies (ergotism and neurofibromatosis), ${ }^{4-7,11,12}$ most of which have specific characteristics that facilitate their diagnosis. ${ }^{4}$

Differential diagnoses should be investigated, as they require specific interventions. ${ }^{3,4}$ Ruling out infectious aortitis should be a priority, since its rapid evolution and poor short-term prognosis make it a therapeutic emergency. ${ }^{4}$ In addition, other rare differential diagnoses should be considered, since their treatment may differ from that of inflammatory aortitis. This may include idiopathic retroperitoneal fibrosis secondary to neoplasia or malignant blood disorders..$^{4-6}$ IgG4-related disease, Erdheim-Chester disease, and inflammatory aneurysms of the abdominal aorta attributable to atherosclerosis are other differential diagnoses to be considered in the presence of aortitis. ${ }^{4}$

The clinical presentation was unusual in the present case because of the young age of the patient, magnitude of the aneurysm, and the lack of typical risk factors associated with AAA development. There was no family history suggestive of underlying connective tissue disease, such as Marfan's syndrome, Ehlers-Danlos syndrome, or Loeys-Dietz syndrome. ${ }^{4,7,11,12}$ However, the clinical history and tests do not meet the criteria established for clinical diagnosis, as genetic tests for relevant mutations are not available at our institution.

Surgical intervention for TA is based on the location and extent of the disease, with aggravated conditions observed in the presence of active inflammation. ${ }^{3}$ Aneurysmatic disease in children and youth with arteritis is extremely rare, with only a few isolated case reports in the literature., ${ }^{3,6,11-13}$ The angiographic types of vascular involvement in TA were defined at an International Conference held in Tokyo in 1994. ${ }^{14}$ According to the distribution of the lesions: Type I: Primary involvement of the branches of the aortic arch. Type IIa: Ascending aorta, aortic arch, and its branches. Type IIb: Ascending aorta, aortic arch and its branches, and descending thoracic aorta. Type III: Descending aorta, abdominal aorta, or renal artery. Type IV: Affects only the abdominal aorta and/or renal arteries. Type V: Combination of IIb and IV. ${ }^{14}$ Thus, the present case report falls into the type IV affection category, which corroborates with the multicentric work carried out in Brazil with 71 young people diagnosed with TA. ${ }^{15}$

Three treatment options are available for AAA patients: conventional open surgery, minimally invasive endovascular repair, or optimized medical treatment based on active surveillance and control of risk factors. ${ }^{3-10}$ The best option depends on such factors as the patient's clinical condition, the anatomical characteristics of AAA, and the presence of symptoms or signs of complications. ${ }^{12,13,16}$

In our patient, drug treatment was started based on a non-selective beta-blocker and statin, in addition to the usual symptomatic medications. She had been using oral glucocorticoid for 2 months and had a history of pulse therapy in a previous hospitalization.

In 2014, the Cochrane Collaboration conducted a meta-analysis to assess the advantages and disadvantages of emergency endovascular repair as compared to the conventional open surgery for the treatment of AAA rupture. They concluded that there was no difference in the mortality rate for up to 30 days between the two treatments. ${ }^{17}$ We have chosen the conventional approach, as materials for an emergency endovascular intervention are not readily available at our institution.

Thus, diagnosing TA is still extremely challenging in view of its nonspecific clinical and laboratory evidence. It is necessary to acknowledge it as a differential diagnosis in vascular diseases, especially in young female patients. This will enable an early intervention and permit an effective modification of its natural history.

Thus, although AAA is unusual in a young population, it should be considered in any patient with abdominal and lumbar pain due to its association with chronic and nonspecific inflammatory diseases. Conventional surgical repair using principles of vascular surgery is the 
treatment of choice in emergencies. Long-term follow-up is essential given the chronicity of the disease.

\section{Author contributions}

Conception and design of the research: Reis JMC, Melo GS. Acquisition of data: Reis JMC, Silva TMMF, Ferreira HLS, Santos FEO. Analysis and interpretation of the data: Reis JMC, Oliveira MV. Writing of the manuscript: Reis JMC, Melo GS, Oliveira MV. Critical revision of the manuscript for intellectual content: Reis JMC, Melo GS, Oliveira MV. Supervision / as the major investigador: Reis JMC.

\section{Potential Conflict of Interest}

No potential conflict of interest relevant to this article was reported.

\section{References}

1. Callicutt CS, Rush B, Eubanks T, Abul-Khoudoud OR. Idiopathic renal artery and infrarenal aortic aneurysms in a 6-year-old child: case report and literature review. J Vasc Surg. 2005;41(5):893-6.

2. Peng NZ, Tze C. Endovascular aneurysm repair (EVAR) of an infra-renal abdominal aortic aneurysm (AAA) in a young patient with Systemic Lupus Erythematosus (SLE). Vasc Med Surg. 2017,5:3.

3. Tavora F, Burke A. Review of isolated ascending aortitis: differential diagnosis, including syphilitic, Takayasu's and giant cell aortitis. Pathology. 2006;38(4):302-8

4. Rousselin C, Pontana F, Puech P, Lambert M. Differential diagnosis of aortitis. 2016;37(4):256-63.

5. Cury M, Zeidan F, Lobato AC. Aortic disease in the young: genetic aneurysm syndromes, connective tissue disorders, and familial aortic aneurysms and dissections. Int J Vasc Med. 2013;2013:267215.

6. Sarkar R, Coran AG, Cilley RE, Lindenauer SM, Stanley JC. Arterial aneurysms in children: clinicopathologic classification. J Vasc Surg. 1991;13:47-57.

7. Washiyama N, Kazui T, Takinami M. Surgical treatment of recurrent abdominal aortic aneurysm in a patient with systemic lupus erythematosus. J Vasc Surg. 2000;32:209-12.

8. Manda GR, Chaziya P, Mwafulirwa W, Kasenda S, Borgstein E. Successful open surgical repair of an infrarenal, abdominal aortic aneurysm (AAA) in a young Malawian female: A case report. Malawi Medical Journal. 2019;31(4): 256-8.

9. Moon SB, Shin WY, Park YJ, Kim SJ. An abdominal aortic aneurysm in an 8- month-old girl with tuberous sclerosis. Eur J Vasc Endovasc Surg. 2009;37:569-71.

\section{Sources of Funding}

There were no external funding sources for this study.

\section{Study Association}

This study is not associated with any thesis or dissertation work.

\section{Ethics approval and consent to participate}

This article does not contain any studies with human participants or animals performed by any of the authors.

\section{Consent}

Written informed consent was obtained the patient for the publication of this case and any accompanying images.

10. Dueppers P, Duran M, Grabitz K, Schelzig H. Open Repair for Abdominal Aortic Aneurysm in a young boy with Tuberous Sclerosis and Review of the Literature. Annals of Vascular Surgery. 2016;1-9.

11. Kuivaniemi H, Tromp G, Prockop DJ. Genetic causes of aortic aneurysms. Unlearning at least part of what the textbooks say. J Clin Invest. 1991;88(5):1441-4.

12. Marconato R, Inzaghi A, Cantoni GM, Zappa M, Longo T. Syphilitic aneurysm of the abdominal aorta: report of two cases. Eur J Vasc Surg. 1988; 2:199-203.

13. Ye C, Yin H, Lin Y, Zhou L. Abdominal aorta aneurysms in children: Single-center experience of six patients. Ann Thorac Surg 2012; 93:201-6.

14. Hata A, Noda M, Moriwaki R, Numano F. Angiographic findings of Takayasu arteritis: New classification. Int J Cardiol. 1996;54 suppl:S155-63.

15. Clemente G, Hilário MO, Len C, Silva CA, Sallum AM, Campos LM et al. Estudo multicêntrico brasileiro de 71 pacientes com arterite de Takayasu juvenil: características clínicas e angiográficas. Rev Bras Reumatol. 2016. http://dx.doi.org/10.1016/j.rbr.2015.09.004.

16. Karthekeyan BR, Vakamudi M, Thangavel P. Repair of idiopathic ascending aortic aneurysm in a 7-year-old child. Cardiol Young. 2015;25(1):154-7.

17. Badger S, Forster R, Blair PH, Ellis P, Kee F, Harkin DW. Endovascular treatment for ruptured abdominal aortic aneurysm. Cochrane Database Syst Rev. 2017;5(5):005261. 